\title{
Diagnosis of Different Electrocardiographic Signals using Recurrent Neural Network and Power Spectrum Analysis
}

\author{
Islam A. Fouad \\ Biomedical Engineering Dept. \\ Misr University for Science and Technology, Giza, Egypt
}

\begin{abstract}
Among the leading causes of death that affect people today are heart problems. It is possible to prevent sudden death by early detection and treatment of heart conditions. Electrical signals from the heart are recorded as electrocardiograms (ECGs). Deaths due to heart diseases comprise a great deal of human mortality. Signals obtained from the ECG, which are easily obtained without damaging the patient, can serve as good indicators of the type of disorder occurring while the heart is operating. Five different abnormal signals have been classified in this study: Atrial fibrillation (AFIB), Atrial premature beats (APB), Pacemaker rhythm (PR), Left bundle branch block (LBBB), and Bigeminy. The classification performances have also been evaluated. Signals are analyzed using time-frequency analysis and signal features are determined by spectral entropy. The applied deep learning algorithm is the Long Short-Term Memory "LSTM" network. With a dataset from twenty eight participants composed of five different categories, a trained Network method achieved an overall accuracy of $97.574 \%$. In the automatic diagnosis of multiple ECG abnormalities, the performance evaluations of the suggested technique convey its robustness and reliability.
\end{abstract}

\section{Keywords}

Electrocardiogram ECG, Signal Analysis, Extracting Features, long short-term memory (LSTM) networks, and Classification

\section{INTRODUCTION}

In order to diagnose cardiological dysfunction, ECGs must be able to detect and classify different types of heartbeats. The clinicians use Holter recorders to record some arrhythmias because they occur at infrequent intervals. Analysis of the recordings of very long Holter ECGs will be facilitated by special algorithms used for the analysis of ECG recordings. In this study, the performance of a classification algorithm was assessed for the classification of five abnormal beats.

There are studies in the literature for the classification of ECG beats[2]. Engin tried to classify ECG pulses using fuzzyhybrid artificial neural networks. In the classification processes, autoregressive model coefficients, high-order cumulant and wavelet transform variances were used as features instead of ECG pulse. For classification, high performance was achieved by using fuzzy c-means algorithm and MLP artificial neural networks[3]. In Erdoğmuş's study,

Discrete Wavelet Transform was applied to the ECG signals obtained from the MIT-BIH database. Daubechies-2 (db2) wavelets from wavelet functions are used. Level 4 CD4 coefficients were used for feature extraction. Artificial neural networks (ANNs) were used for classification and four groups of feature coefficients were tested. Although normalization and QRS detection were not performed in the test results, it was stated that the classification success was higher than the Fast Fourier Transform[4]. Yu used independent component analysis (ICA) and artificial neural networks to classify ECG pulses in his study. ICA was used to calculate statistically mutually independent principal components of ECG signals. $\mathrm{RR}$ interval and their projections combine to form the feature vector of the following classifier. Classifiers were built using probabilistic neural networks (PNNs) and feedback artificial neural networks (BPNNs) [5]. An artificial neural networkbased model was developed by Güler to classify ECG pulses in his study. A discrete wavelet transform (DWT) is used to discretize ECG signals into time-frequency domains and to calculate their statistical properties. The classification of ECG pulses has been achieved using first-level networks, which are based on statistical features. Using the dislocation of firstlevel networks as input data, second-level networks are trained to improve diagnostic accuracy. $96.94 \%$ accuracy was achieved by classifying ECG beats from the Physiobank database (Normal beats, congestive heart failure beats, ventricular tachyarrhythmia beats, atrial fibrillation beats) with the combined network model[6].

This paper presents a method for pre-processing and categorizing abnormalities, based on features extracted from the ECG signals, which is then used to train an artificial neural network. It discusses five types of abnormal ECG patterns.

Atrial fibrillation (AFIB), Atrial premature beats (APB), Pacemaker rhythm (PR), Left bundle branch block (LBBB), and Bigeminy are the investigated abnormal ECG signals.

Next, the manuscript will use the abbreviations presented by table (1).

Table 1. Abnormalities' Anonymous

\begin{tabular}{lll}
\hline No. & Full Name of ECG disease & Used Abbreviation \\
\hline $\mathbf{1}$ & Atrial fibrillation & AFIB \\
$\mathbf{2}$ & Atrial premature beats & APB \\
$\mathbf{3}$ & Pacemaker rhythm & PR \\
$\mathbf{4}$ & Left bundle branch block & LBBB \\
$\mathbf{5}$ & $\begin{array}{l}\text { Premature ventricular } \\
\text { contraction }\end{array}$ & Bigeminy \\
\hline
\end{tabular}


In figure 1, samples of five abnormalities tested are shown based on datasets downloaded from the data verse website.

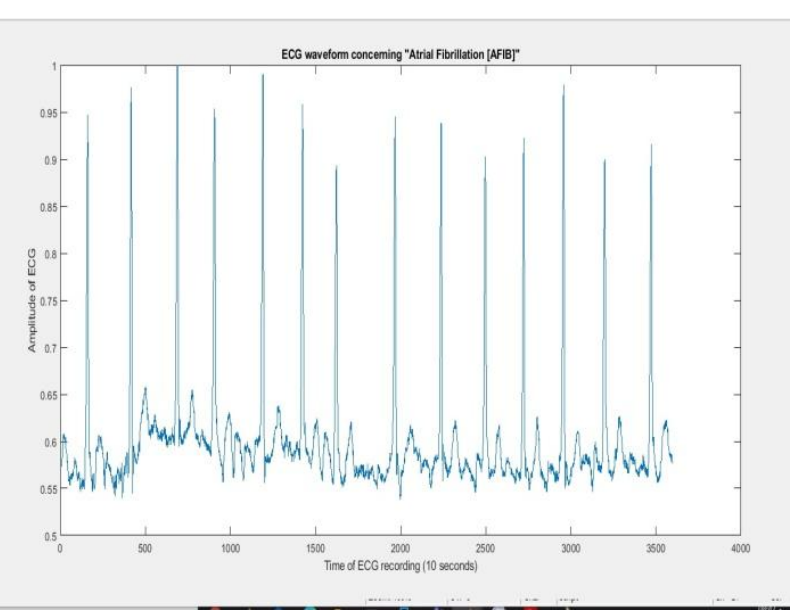

a. Atrial fibrillation - AFIB
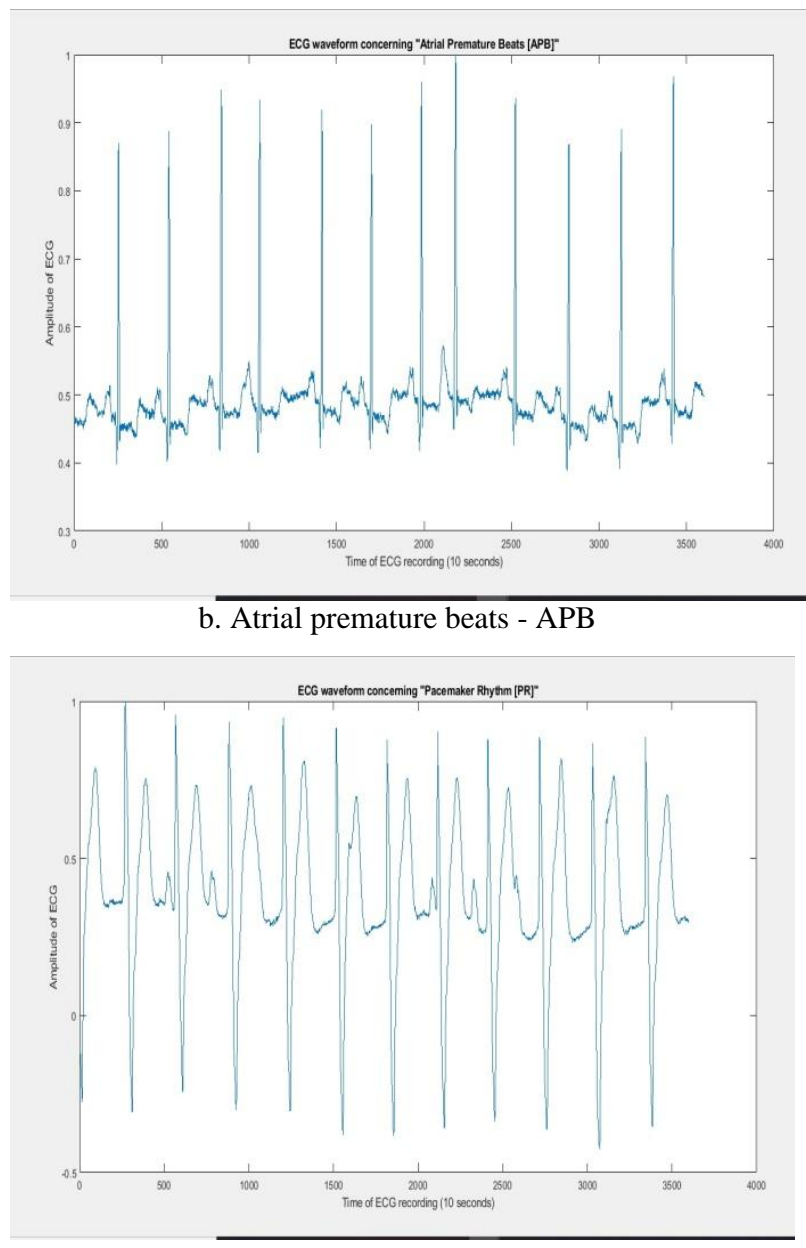

c. Pacemaker rhythm - PR

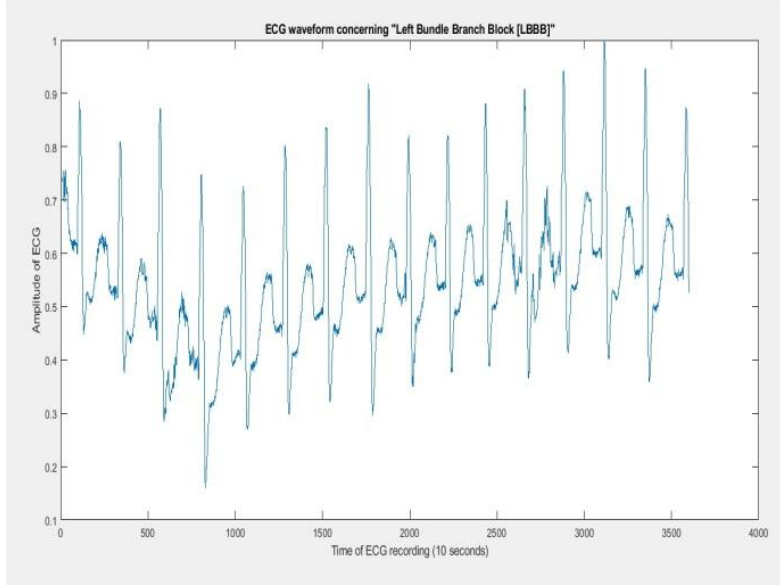

d. Left bundle branch block - LBBB

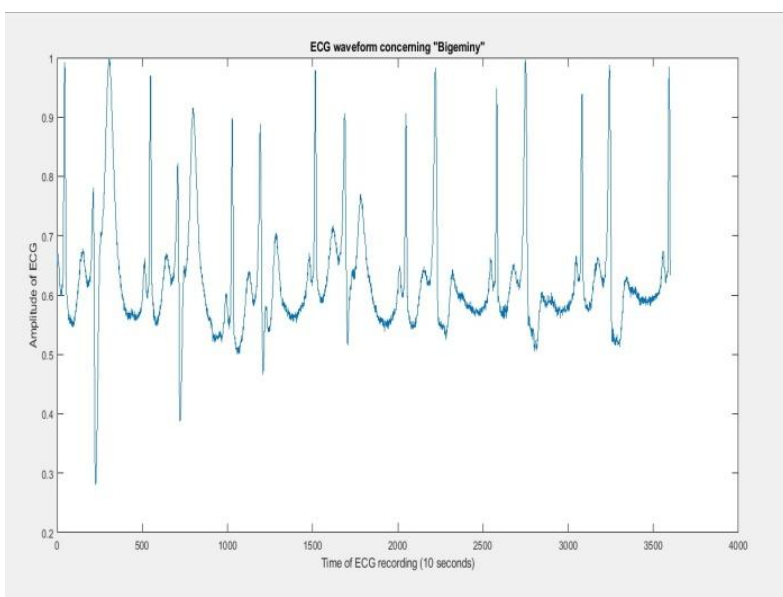

e. Bigeminy

Figure 1, Samples extracted from the dataset

\section{MATERIALS}

The MATLAB platform, in conjunction with its Deep Learning and Signal Processing Toolboxes, is an efficient resource for signal-based tools used for medical diagnosis. The Signal Processing Toolbox is used to process the signals and evaluate their features, while the Deep Learning Toolbox is utilized for training a system of LSTM Network, as it is utilized for classifying signals into one of the five classes.

About 313 ECG vectors were collected from 28 patients. The dataset was downloaded from the PhysioNet service (http://www.physionet.org) from the MIT-BIH Arrhythmia database [7] and table 2 shows its description.

Table 2. Full description of the dataset

\begin{tabular}{cccl}
\hline Dataset & $\begin{array}{c}\text { Total } \\
\text { Number } \\
\text { of } \\
\text { Signals }\end{array}$ & $\begin{array}{c}\text { Format } \\
\text { of } \\
\text { Signal }\end{array}$ & No. of Abnormal Signals \\
\hline $\begin{array}{c}\text { Pawel } \\
\text { Plawiak } \\
(\mathbf{2 0 1 9 )}\end{array}$ & 313 & MAT & $\begin{array}{l}\text { Atrial fibrillation (93 } \\
\text { vectors), } \\
\end{array}$ \\
& & Files & Atrial premature beats (58 \\
& & & vectors) \\
& & Pacemaker rhythm (30 \\
\hline
\end{tabular}


vectors)

Left bundle branch block ( 88 vectors)

Bigeminy (44 vectors)

Dataset was divided into training and testing groups, corresponding to $40 \%$ and $60 \%$ of the total dataset respectively.

So; 125 vectors were selected as training dataset and the remaining 188 vectors for testing purpose.

The specifications of the used $\mathrm{PC}$ on which the programs were designed are : Intel(R) Core(TM) i5-2450M CPU running at $2.50 \mathrm{GHz}$ processor with $8 \mathrm{~GB}$ RAM. Program coding was made using the high performance and powerful language "MATLAB" version 9.10.0.1602886 (R2021a). The following toolboxes: "signal processing", and "wavelet transform" which supports an extensive range of very important operations were utilized for technical computing and data analysis.

\section{METHODS}

\subsection{ECG Data}

The normal ECG signal consists of certain waves called P, Q, $\mathrm{R}, \mathrm{S}$, and $\mathrm{T}$, which are aligned on the baseline level of the heart at rest. Sometimes a small amplitude U wave may follow the $\mathrm{T}$ wave. The part called the $\mathrm{P}$ wave is formed as a result of contraction of the atria. The PQ interval represents the transmission time of the his beam. The QRST wave is called the ventricular complex. QRS means ventricular depolarization. Conduction defects in the bundle of His and its arms cause changes in the QRS wave[8].

As Shown in Figure (2); normal heartbeat includes basic parameters such as wave shape, duration, RR interval. Changes in these parameters indicate a heart condition. This irregularity in the ECG signal is called arrhythmia and arrhythmias are dangerous for the patient[1], [8].

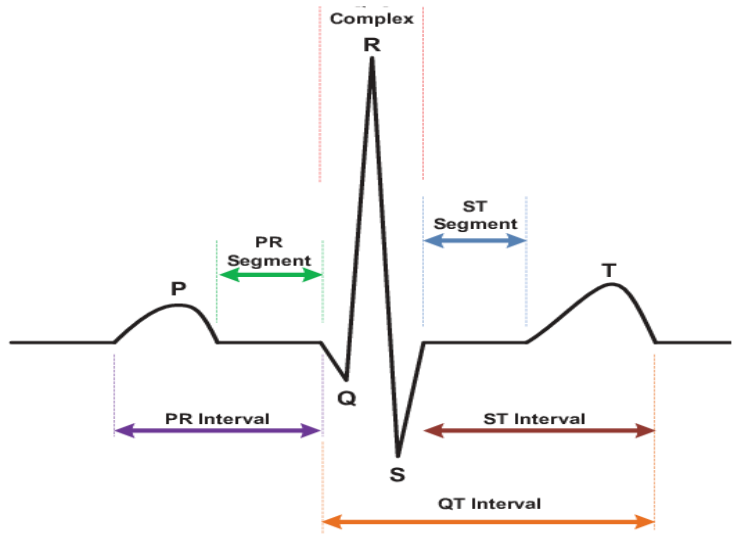

Figure 2, Significant waves in a normal ECG beat

\subsection{Feature Extraction}

The concept of entropy can be defined as a mathematical measure of uncertainty and disorder about an event. It emerged in 1948 with Claude Elwood Shannon, an electronic engineer and mathematician [9].

Entropy can be calculated as :

$$
\mathrm{H}(\mathrm{x})=\sum_{i=1}^{n} \mathrm{p}(\mathrm{Xi}) \log [\mathrm{p}(\mathrm{Xi})]
$$

Here, $p(X i)$ is the possible probabilities and $\mathrm{N}$ is the signal length.

Spectral Entropy (SpEn) is the entropy that determines the degree of regularity in complex signals. A signal with uniform probability values will have high entropy, and a signal with irregular probability values will have low entropy. Unlike entropy, power calculates the probability value of the spectral density [10].

It's calculated as:

$$
\operatorname{SpEn}=\sum_{f}\left(\mathrm{P}(\mathrm{f}) \log \frac{1}{P(f)}\right)
$$

\subsection{Classifying the Disease}

It is difficult to accurately classifying abnormalities to their proper classes when the number of classes increase. Many research have shown that using Neural Networks are highly performed when applied in medical diagnostic systems [11] [12]. In this paper, "Long-Short Term Memory" Network (RNN) classification method is performed.

LSTM is an RNN-based deep learning method. It has a learning architecture that can remember long-term dependencies at random intervals. It is a very successful method especially in analyzing incoming data sequentially according to time or events with a certain relationship [13]. With $\mathrm{x}=\left\{\mathrm{X}_{1}, \cdots, \mathrm{X}_{\mathrm{T}}\right\}$ as an ordinal data, $\mathrm{RNN}$ follows the hidden vector sequence $\mathrm{h}=\left\{\mathrm{h}_{1}, \cdots, \mathrm{h}_{\mathrm{T}}\right\}$ and $\mathrm{y}=\left\{\mathrm{y}_{1}, \cdots, \mathrm{y}_{\mathrm{T}}\right.$ \}. It finds the order of the output vector proceeding as this calculation is done with $\mathrm{T}$ iterations

It is as follows:

$$
\begin{gathered}
h_{\mathrm{t}}=\mathrm{H}\left(W_{x h} x_{t}+W_{h h} h_{t-1}+b_{h}\right) \\
y_{\mathrm{t}}=F\left(W_{h y} h_{t}+b_{y}\right)
\end{gathered}
$$

The $W_{x h}, W_{h h}$, and $W_{h y}$ matrices are the connection weights calculated at training time. $F$ is the sigmoid activation function.

The sigmoid is defined as follows:

$$
F=\frac{e^{z m}}{\sum_{k} e^{z k}}
$$

\section{RESULTS AND DISCUSSIONS}

A dataset of 330 ECG vectors was used for the experimental analysis. The dataset was decomposed into training and testing, each set contained $40 \%$, and $40 \%$ of the total dataset, respectively.

The neural network was trained independently using different training methods. Then, calculating its sensitivity, accuracy, and specificity. Sensitivity was calculated from the proportion of positives that were distinguished. Specificity, on the other hand, was calculated from the proportion of negatives that were distinguished. Specificity and sensitivity formulas were obtained as follows: specificity

Sensitivity 
Table (2) shows the TP, TN, FP, FN, while table (3) and figure (3) present the accuracy, sensitivity, specificity, precision, and F_score of the different five classes after classification of 330 heart diseases' vectors.

Table 2. TP, TN, FP, and FN results of the proposed technique

\begin{tabular}{lllll}
\hline & TP & TN & FP & FN \\
\hline AFIB & 289 & 34 & 4 & 3 \\
APB & 34 & 291 & 2 & 3 \\
PR & 4 & 323 & 2 & 1 \\
LBBB & 301 & 14 & 9 & 6 \\
Bigeminy & 222 & 98 & 8 & 2 \\
\hline
\end{tabular}

Table 3. Acc., Sens., Prec., and F_score results of the proposed technique

\begin{tabular}{crrrr}
\hline & Accuracy & Sensitivity & Precision & F_Score \\
\hline AFIB & $97.88 \%$ & $98.97 \%$ & $98.63 \%$ & $98.80 \%$ \\
APB & $98.48 \%$ & $91.89 \%$ & $94.44 \%$ & $93.15 \%$ \\
PR & $99.09 \%$ & $80.00 \%$ & $66.67 \%$ & $72.73 \%$ \\
LBBB & $95.45 \%$ & $98.05 \%$ & $97.10 \%$ & $97.57 \%$ \\
Bigeminy & $96.97 \%$ & $97.80 \%$ & $96.52 \%$ & $97.80 \%$ \\
\hline
\end{tabular}

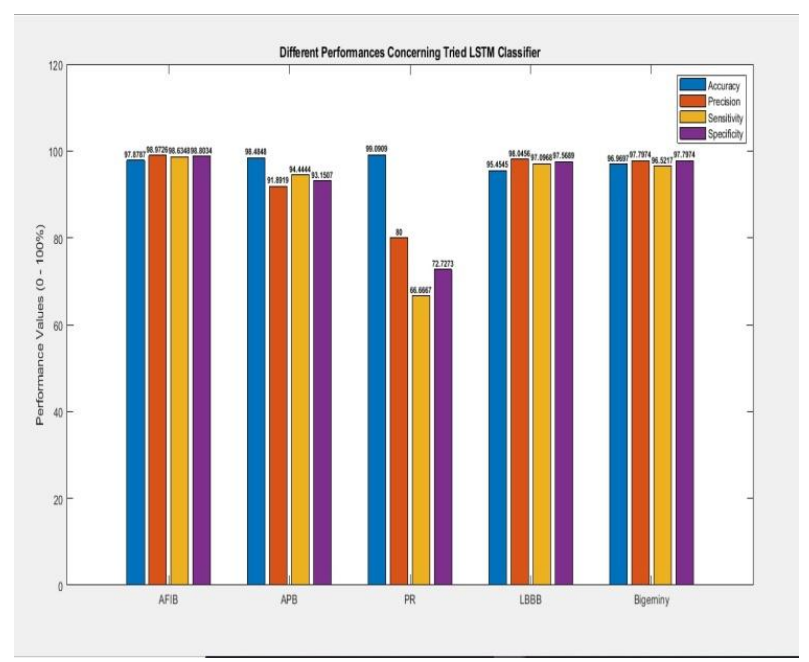

Figure 3, The Acc., Sens., Prec., and F_score results of the proposed technique
According to Figure (3) and the confusion matrix, an overall classification accuracy of $97.574 \%$ was achieved and there is no misclassification concerning any of the five classes.

Tables (4) and (5) show a comparison between the presented work and some of the literature.

Table 4. Comparison of results with literature (Part 1)

\begin{tabular}{cccc}
\hline Researcher & Features & Classifier & $\begin{array}{c}\text { Training Data } \\
\text { Test Data }\end{array}$ \\
\hline Hu [14] & $\begin{array}{c}\text { Interval } \\
\text { Morphology }\end{array}$ & $\begin{array}{c}\text { SOM+LVQ } \\
\text { KL-PCA, } \\
\text { AAMI }\end{array}$ & $\begin{array}{c}15 \text { records Train } \\
\text { 20 records Test }\end{array}$ \\
Chazal [15] & Amplitudes & Hybrid LD & 22 records Train \\
& Intervals & Lead A + lead B & 22 records Test \\
Chazal [16] & Amplitudes & Hybrid LD & DSI + 500 beats \\
& Intervals & general + & 22 records \\
Park [17] & RR Intervals & SVM & Train 22 Rec \\
& HOS and & & (30 min) \\
& HBF & & Test 22 Rec (30 \\
& min) \\
This Study & Spectral & LSTM Network & 132 records \\
& Entropy & & Train \\
& & & 198 records Test \\
\hline
\end{tabular}

Table 5. Comparison of results with literature (Part 2)

\begin{tabular}{ccc}
\hline Researcher & Training Scheme & Results Se, PPV \\
& & \\
\hline Hu [14] & First 5-min & Overall Se\% 82.6 \\
& Patient-specific & Overall PPV\% 77.7 \\
Chazal [15] & AAMI & $86.8,75.9,77.7,89.4$ \\
& Inter Patient & $99.1,38.5,81.5,8.6$ \\
Chazal [16] & AAMI & $94.2,87.7,94.3,73.9$ \\
& Patient-specific & $99.3,46.9,94.3,29.1$ \\
Park [17] & AAMI & $86.3,82.6,80.9,54.9$ \\
& Inter Patient & \\
This Study & 132 records Train & $97.574 \%$ \\
\hline
\end{tabular}

\section{CONCLUSION}

In this study, a processing way for classifying different heart diseases into their particular groups using one of the artificial recurrent neural networks (RNN) was presented. Recurrent Neural Networks are an advanced approach for complex data classification with high performance [18]. The tried 330 ECG records were classified into their particular groups, with a total accuracy of $97.574 \%$, using "Spectral Entropy" as the discriminating feature.

After training the network on a large and various dataset with high intra-class variability, the total accuracy rate and the system's performance was improved. This was reflected in reducing misclassification and increasing accuracy. Increasing the number of features extracted from signal is another way to diversify the dataset.

From these findings, future work will focus on improving classification results and the total accuracy. The number of output classes can be maximized as well.

Future work will focus on increasing the output classes while improving the results and overall accuracy and trying different datasets as more data becomes available. 


\section{REFERENCES}

[1] Z. Dokur, "Classification of ECG Pulses Using Artificial Neural Networks and Genetic Algorithms," Istanbul Technical University, 1999.

[2] G. Bortolan, I. Jekova, and I. Christov, "Comparison of four methods for premature ventricular contraction and normal beat clustering," in Computers in Cardiology, 2005, 2005, pp. 921-924.

[3] M. Engin, "ECG beat classification using neuro-fuzzy network," Pattern Recognit. Lett., vol. 25, no. 15, p. 1715-1722, Nov. 2004.

[4] P. Erdoğmuş and A. Pekçakar, "Feature extraction of ECG signals with wavelet transform and classification with artificial neural networks," pp. 13-15, 2009.

[5] S. Yu and K. Chou, "Integration of independent component analysis and neural networks for ECG beat classification," Expert Syst. Appl., vol. 34, pp. 28412846,2008

[6] Güler and E. D. Übeylı", "ECG beat classifier designed by combined neural network model," Pattern Recognit., vol. 38, no. 2, p. 199-208, Feb. 2005.

[7] http://www.physionet.org and https://ieeedataport.org/documents/ecg-signals-744-fragments\#files

[8] E. Yazgan and M. Korurek, Medical Electronics, 1st Ed. Istanbul: Istanbul Technical University Offset Printing Workshop, 1996

[9] Shannon, C. E. (1948). Communication theory of secrecy systems. Bell system technical journal, 28(4):656-715.

[10] Sabeti, M., Katebi, S. ve Boostani, R. (2009). Entropy and complexity measures for EEG signal classification of schizophrenic and control participants. Artificial Intelligence in Medicine, 47: 263-274
[11] Andre Esteva, Brett Krupel and Sebastian Thrun, Deep Networks for Early Stage Skin Disease and Skin Cancer Classification, Stanford University, 2015.

[12] Masood A., Al-Jumaily A.A., Adnan T., Development of Automated Diagnostic System for Skin Cancer: Performance Analysis of Neural Network Learning Algorithms for Classification, Wermter S. et al. (eds) Artificial Neural Networks and Machine Learning ICANN 2014. ICANN 2014. Lecture Notes in Computer Science, vol 8681. Springer, Cham

[13] Sepp Hochreiter, Jürgen Schmidhuber, "Long Short-term Memory", Neural Computation 9(8):1735-80, December 1997.

[14] Hu, Y.H., Palreddy, S., Tompkins, W.J., 1997. A patientadaptable ecg beat classifier using a mixture of experts approach. IEEE transactions on biomedical engineering $44,891-900$.

[15] De Chazal, P., O’Dwyer, M., Reilly, R.B., 2004 Automatic classification of heartbeats using ecg morphology and heartbeat interval features. IEEE transactions on biomedical engineering 51, 1196-1206.

[16] De Chazal, P., Reilly, R.B., 2006. A patient-adapting heartbeat classifier using ecg morphology and heartbeat interval features. IEEE transactions on biomedical engineering $53,2535-2543$

[17] Park, K., Cho, B., Lee, D., Song, S., Lee, J., Chee, Y., Kim, I., Kim, S., 2008. Hierarchical support vector machine based heartbeat classification using higher order statistics and hermite basis function, in: 2008 Computers in Cardiology, IEEE. pp. 229-232.

[18] Islam A. Fouad, Hany Elnashar, "An Efficient and HighPerformance System for Skin Cancer MultiClassification Using Machine Learning", Annals of RSCB, vol. 25, no. 6, pp. 20931-20945, Nov. 2021. 